\title{
Acute surgical abdomen during the COVID-19 pandemic: Clinical and therapeutic challenges
}

\author{
DRAGOS SERBAN ${ }^{1,2^{*}}$, BOGDAN SOCEA $^{1,3^{*}}$, CRISTINEL DUMITRU BADIU $^{1,4}$, \\ CORNELIU TUDOR $^{2}$, SIMONA ANDREEA BALASESCU ${ }^{2}$, DAN DUMITRESCU ${ }^{1,2}$, ANDRA MARIA TROTEA ${ }^{1,5}$, \\ RADU IULIAN SPATARU ${ }^{1,6}$, GETA VANCEA ${ }^{1,7}$, ANA MARIA DASCALU ${ }^{1}$ and CIPRIAN TANASESCU ${ }^{8 *}$
}

\begin{abstract}
${ }^{1}$ Faculty of Medicine, 'Carol Davila' University of Medicine and Pharmacy, 020021 Bucharest; ${ }^{2}$ Fourth Department of Surgery, Emergency University Hospital of Bucharest, 050098 Bucharest; ${ }^{3}$ Department of Surgery, 'Sf. Pantelimon' Emergency Hospital, 021659 Bucharest; ${ }^{4}$ General Surgery, Emergency Clinical Hospital 'Prof. Dr. Bagdasar Arseni', 041915 Bucharest; ${ }^{5}$ Second Department of Anesthesia and Intensive Care, Emergency University Hospital of Bucharest, 050098 Bucharest; 'Department of Paediatric Surgery, Emergency Clinic Hospital for Children 'Maria S. Curie', 41451 Bucharest; ${ }^{7}$ Third Department, 'Dr Victor Babes' Clinical Hospital for Infectious and Tropical Diseases, 030303 Bucharest; ${ }^{8}$ Third Department of Surgery, Faculty of Medicine, 'Lucian Blaga' University Sibiu, 550169 Sibiu, Romania
\end{abstract}

Received January 18, 2021; Accepted February 17, 2021

DOI: $10.3892 / \mathrm{etm} .2021 .9950$

\begin{abstract}
The present study investigated the effects of the COVID-19 pandemic on the clinical presentation and therapeutic management of acute surgical abdomen. A retrospective study of emergency hospitalizations with a diagnosis of acute surgical abdomen between April and July 2020 vs. a similar period in 2019 was performed. The observation sheets and the operating protocols were analyzed. Between April and July 2020, 50 cases of acute surgical abdomen were hospitalized and treated, compared to 43 cases in the same period last year. The main types of pathology in both groups included: Occlusions (60\%, respectively $44.2 \%$ in 2019$)$ and peritonitis (32\%, respectively $41.8 \%$ in 2019). There was an increased rate of patients with colorectal cancers neglected therapeutically or uninvestigated, who presented during the pandemic period with emergencies for complications such as occlusion or tumor perforation (32 vs. 6.97\%, $\mathrm{P}=0.0039$ ). One case, with gastric perforation, was COVID-positive, with no pulmonary symptoms at admission. The number of postoperative infectious complications was lower during the pandemic ( 2 vs. $13.95 \%$, P=0.0461). As the COVID-19 pandemic appears to be still far from ending, we should learn to adapt our surgical protocols to the new evidence.
\end{abstract}

Correspondence to: Dr Bogdan Socea, Faculty of Medicine, 'Carol Davila' University of Medicine and Pharmacy, 37 Dionisie Lupu Street, 020021 Bucharest, Romania

E-mail: bogdan.socea@umfcd.ro

*Contributed equally

Key words: emergency surgery, COVID-19 pandemic, acute abdomen, oncological
Oncological patients are a vulnerable group, who were neglected in the first months of the pandemic. SARS-Cov-2 infection may be a cause of abdominal pain and should be taken into account in different diagnoses of acute abdomen in surgical wards. Correct wearing of adequate personal protective equipment (PPE) and respecting strict rules of asepsis and antisepsis are required for preventing in-hospital transmission of infection.

\section{Introduction}

The COVID-19 pandemic has emerged as a significant threat to healthcare systems, and treatment algorithms have changed in general surgical clinics, as has been the case in other medical disciplines during the outbreak. Although comorbidities and advanced age are factors statistically associated with increased mortality, the existence of severe forms of COVID-19 in young adults and among medical staff has increased the psychological pressure of those treating patients suspected or infected with SARS-CoV-2 (1-4).

The etiologic agent, SARS-Cov-2, is an RNA virus in the Coronavirus family, generally responsible for benign respiratory infections, except for the causative agents of the Middle East respiratory syndrome (MERS) in 2012 and severe acute respiratory syndrome (SARS) during the 2002-2003 outbreaks (3-5). Infection modalities confirmed by clinical trials are through droplets, aerosols, direct and indirect contact $(6,7)$. To prevent in-hospital contamination of medical personnel, the new protocol guidelines recommend avoiding all medical-surgical procedures with the potential to generate aerosols in suspected infected individuals or whose COVID-19 status has not yet been evaluated, including endoscopy and laparoscopy, and performing them with appropriate protective equipment, only when the benefit to the patient outweighs the risk (8-16). This has a significant impact on the management of acute abdomen, a clinical entity of special severity, whose 
investigation and surgical approach does not allow a time delay until obtaining the result of RT-PCR testing for SARS-Cov-2.

The present study investigated the effects of the COVID-19 pandemic on the clinical presentation and therapeutic management of acute surgical abdomen.

\section{Patients and methods}

Study design. We conducted a retrospective comparative study on the emergency hospitalizations for patients diagnosed with acute surgical abdomen at the Fourth Department of General Surgery, Emergency Hospital Bucharest, Romania, between April and July 2020 vs. the similar period last year (2019).

Clinical presentation, etiology, diagnostic, paraclinical investigations and therapeutic management, as well as the changes in emergency practice procedures imposed by the COVID-19 pandemic were analyzed.

The inclusion criteria were patients admitted to the emergency room (ER), surgical or managing conservatory with one of the following diagnoses: Bowel obstruction, peritonitis, superior/inferior digestive hemorrhage or acute mesenteric ischemia. Patients who refused hospitalization or with incomplete data were excluded from the statistical analysis.

Data analysis. The data were obtained from the ER register, the observation sheets and the operating protocols. For descriptive analysis, the medians, means, and standard deviations were used. Fischer test and t-test (SPSS 13.0 version; SPSS Inc.) were used to assess statistically significant correlations between the two study groups in terms or age, sex, etiopathology, severity of symptoms at admission and therapeutic approach. The study was not subject to formal consent of the ethics boards of the participating hospitals due to its retrospective nature.

\section{Results}

Between April and July 2020, during the COVID-19 pandemic, 50 patients diagnosed with acute surgical abdomen were hospitalized and treated, compared to 43 cases in the same period in 1999. There were no statistically significant differences between the two groups of patients in terms of average age and sex ratio. The percentage of male patients was slightly higher during the pandemic period (Table I).

The main types of hospitalized pathology for acute abdomen were, in both groups: Occlusions (60\%, respectively $44.2 \%$ in 2019) and peritonitis (32\%, respectively $41.9 \%$ in 2019).

There was an increased rate of patients with colorectal cancers neglected therapeutically or uninvestigated, who presented during the emergency pandemic for complications such as occlusion or tumor perforation (32 vs. $6.97 \%$, $\mathrm{P}=0.0101)$. Informed consent was obtained, especially in these cases, with specific explanations of the possible consequences of the surgical procedure, including temporary or permanent colostomy, an aspect considered possibly disabling to the patient. The therapeutic viewpoint in these cases was to solve the complication by minimal manipulation: (ileo/colostomy) or Hartmann procedure, with tumor removal, if this was possible within the limits of oncological safety, and protective colostomy. In cases with perforation associated with peritonitis as a general rule, colostomy was performed after surgical resolution and a digestive anastomosis was avoided.

A particular challenging case was that of a relatively young patient, without associated pathology, who denied recent trauma, with biochemical and imaging results within normal limits, but who presented with diffuse abdominal pain. Clinical examination showed a tachycardic pulse $(96 / \mathrm{min})$ and mild hypotension $(120 \mathrm{mmHg})$. The patient was admitted to the surgical clinic and investigated surgically. A diagnosis of mesenteric rupture was established. After surgical treatment by segmental enterectomy, the evolution was favorable.

One case was COVID-19 positive, with no pulmonary symptoms at admission. He was treated for gastric perforation, ulcer excision and suture with omentoplasty and after confirmation of SARS-Cov-2 infection was transferred to a COVID-19 support hospital.

Proper handling of personal protective equipment (PPE) and compliance with hygiene rules prevented contamination of staff and other hospitalized patients. The number of postoperative infectious complications was much lower during the pandemic ( 2 vs. $13.95 \%, \mathrm{P}=0.0461$ ). One explanation would be the strict observance of the rules of asepsis and antisepsis and the social distancing in the hospital rooms imposed by the new protocols.

Our surgical protocol during the COVID-19 pandemic. In a hospital with $>1,000$ beds, with multiple medical and surgical specializations, which treats groups of patients with increased vulnerability (oncological, hematological, chronic dialysis patients), with vertical design, it is important to separate staff caring for COVID-19 suspects or confirmed patients and those caring for patients with negative RT-PCR for SARS-Cov 2, to prevent/reduce the risk of in-hospital transmission.

Since the declaration of the COVID-19 pandemic in our country, on March 15, all patients presenting at the ER are treated as potential COVID-19 suspects. Initial examination on the ward is made using complete PPE, with N95 or FFP 2 or 3 (filtering facepiece) masks, eye protection, gowns and gloves. If admission is considered necessary and the patient signs an informed consent for further diagnosis and treatment procedures, RT-PCR is performed from the nasopharyngeal exudate to investigate the presence of SARS-Cov-2 infection. The patient is next transported to the ward following separate circuits for COVID-19 suspects. Until the COVID-19 test result is obtained ( 24-36 h), these patients are assessed in isolated areas. Patients are required to wear masks during the hospital stay.

Medical management is initiated immediately, depending on the case. According to the recommendations of the national and international societies of surgery and endoscopic surgery [Society of American Gastrointestinal and Endoscopic Surgeons (SAGES), European Association for Endoscopic Surgery (EAES), Endoscopic and Laparoscopic Surgeons of Asia (ELSA), Romanian Society of Digestive Endoscopy and Romanian Association of Endoscopic Surgery (SRED-ARCE)], aerosol-generating procedures must be avoided, whenever possible, until COVID infection is confirmed, as a preventive measure of in-hospital contamination with SARS-Cov 2 (8-12). 
Table I. Presentation, management and outcomes of patients with acute abdomen during April-July, 2020 vs. April-July, 2019.

\begin{tabular}{|c|c|c|c|}
\hline & April-July 2020 & April-July 2019 & Fisher test $(\mathrm{P}<0.05)$ \\
\hline Total number & 50 & 43 & 0.9561 \\
\hline Sex ratio $(\mathrm{F}: \mathrm{M})$ & $1: 2.33$ & $1: 1.38$ & 0.2753 \\
\hline Age $($ mean $\pm \mathrm{SD})$ & $60.9 \pm 2.34$ & $61.2 \pm 2.76$ & $>0.999$ \\
\hline \multicolumn{4}{|l|}{ Pathology } \\
\hline Occlusions: & $30(60 \%)$ & $19(44.2 \%)$ & 0.1486 \\
\hline Strangulated hernia/eventration & 9 & 11 & 0.4513 \\
\hline Adherences & 5 & 4 & $>0.999$ \\
\hline Volvulus & 3 & 2 & $>0.999$ \\
\hline Tumor stenosis & 10 & 1 & $0.0095^{\mathrm{a}}$ \\
\hline Simple mechanical occlusion & 3 & 1 & 0.6211 \\
\hline Peritonitis: & $16(32 \%)$ & $18(41.9 \%)$ & 0.3899 \\
\hline Gastric perforation & 2 & 3 & 0.9862 \\
\hline Caecal tumor perforation & 5 & 2 & 0.4447 \\
\hline Appendicitis & 8 & 8 & $>0.999$ \\
\hline Biliary peritonitis & 1 & 0 & $>0.999$ \\
\hline Perforated diverticulitis & 0 & 3 & 0.0951 \\
\hline Crohn's disease & 0 & 1 & $>0.999$ \\
\hline Mesenteric ischemia & 1 & 2 & $>0.999$ \\
\hline Hemorrhages & 3 & 1 & 0.6211 \\
\hline Upper digestive bleeding & 2 & 1 & $>0.999$ \\
\hline Traumatic mesenteric rupture & 1 & 0 & $>0.999$ \\
\hline Neoplastic patients & $16(32 \%)$ & $4(9.3 \%)$ & $0.0101^{\mathrm{a}}$ \\
\hline \multicolumn{4}{|l|}{ Management: } \\
\hline Surgery & $40(80 \%)$ & $38(88.4 \%)$ & $>0.999$ \\
\hline Laparoscopy vs. open surgery & $2 / 38$ & $9 / 29$ & $0.0234^{\mathrm{a}}$ \\
\hline Conservative & $10(20 \%)$ & $5(11.6 \%)$ & 0.3976 \\
\hline \multicolumn{4}{|l|}{ Surgery: } \\
\hline Immediate & 38 & 35 & 0.9862 \\
\hline$>24 \mathrm{~h}$ & 2 & 3 & \\
\hline COVID-19 status (RT-PCR) & & $\mathrm{N} / \mathrm{A}$ & \\
\hline Positive & 1 & & \\
\hline Negative & 49 & & \\
\hline Hospital stay (days) & $8.73 \pm 0.67$ & $10.27 \pm 1.21$ & \\
\hline \multicolumn{4}{|l|}{ Postoperative complications } \\
\hline Deceased & $6(12 \%)$ & $4(9.3 \%)$ & 0.7476 \\
\hline Nosocomial infections: & $1(2 \%)$ & $6(13.95 \%)$ & $0.0461^{\mathrm{a}}$ \\
\hline Surgical wound infection & 1 & 4 & \\
\hline Clostridium infection & 0 & 2 & \\
\hline
\end{tabular}

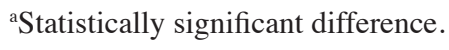

If the condition involves immediate emergency surgery, the patient is transported by passing through a special circuit for COVID-19 suspects. If oxygen is needed, the oxygen masks are placed over the surgical ones $(6-8 \mathrm{l} / \mathrm{min})$. Both anesthetic team and surgical team must wear full PPE with water-resistant gown, mask (KN95 or higher respiratory) and cap, eye protection and double pair of gloves. Only the anesthesiologist and the designated nurse for intubation remain in the room during tracheal intubation and extubating, which are considered the highest risk procedures for transmission of COVID-19, due to aerosolization (15). A high quality viral filter is used between the breathing circuit and the patient's airway and a second filter is placed on the expiratory limb, where it connects to the anesthesia machine. A rapid sequence induction (RSI) and intubation are used: First the stomach content is aspirated on a nasogastric tube, nasal prongs are placed under a surgical mask, two intravenous lines with running fluids are achieved, and preoperative i.v. antacides, 
atiemetics, antibiotics are administrated. After sedation, the anesthetist intubates the patient, inflates the cuff and attaches the filter before connecting to the ventilation machine, and clamps the tube if disconnection is required.

Positive pressure in the operating room is off. The operation is performed by open surgical approach, avoiding laparoscopy in patients who could not be delayed until obtaining the RT-PCR result, according to initial ARCE recommendations, to avoid possible aerosolization of viral particles, in the absence of a pneumoperitoneum filtration system (8-12,16-18). This has led to a significant decreased use of laparoscopy in emergency during the COVID-19 pandemic $(\mathrm{P}=0.0234)$. In addition, the use of the electrocautery is limited, with the setting of a low energy level.

If after surgery the patient is stable and the arterial blood gas tests are good, he is wakened and trasported with a surgical mask with an oxygen mask on it. If the patient remains intubated, the endotracheal tube is clamped for five seconds and then connected to the transport ventilator (with filter), or a high quality heat and moisture exchanging (HME) filter should be inserted between the self-inflating bag and endotracheal tube. The patient is then transported directly to an airbone infection isolation room for recovery. The gas sampling tube is changed after each suspect COVID-19 patient. All trash and linen are placed in red biohazard bags. Special care is taken when dismantling and discarding the circuit from the anesthesia machine. The ultraviolet light disinfection is set on its normal cycle and is run in the OR room to additionally disinfect the room for $30 \mathrm{~min}$, and then the room is cleaned with special disinfectants based on peroxide and another 30 min cleaning pump, until all aerosols have been removed.

\section{Discussion}

The diagnosis of acute surgical abdomen requires immediate life-saving surgical treatment. The clinical entities that fall under this incidence are occlusions, peritonitis, intra-abdominal hemorrhages or externalized at the level of the digestive tract, and entero-mesenteric ischemia, all with a severe clinical course, sometimes even fatal in the absence of surgical resolution (15-18). The clinical presentation of acute surgical abdomen did not change significantly during the COVID-19 pandemic, the only difference encountered was the increased number of oncological patients presented during COVID-19 with acute complications.

Diagnostic challenges in acute surgical abdomen. The difficulty does not lie in the surgical attitude of immediately approaching the patient, exploring, confirming, and solving or even refuting this diagnosis, but in establishing the correct indication for surgery in conditions of discrete or discordant symptoms compared to laboratory data, which can have major, medical, but also legal consequences (19). If an exploratory laparotomy is not desirable, but not imputable, as an extreme measure for confirmation of an uncertain diagnosis, late intervention or absence of surgery can lead to patient death within hours.

The practice has shown that most patients in the emergency department follow a clinical-laboratory protocol, which requires subsequent specialized examinations, surgical ones including, but only a small number represent a surgical emergency. The challenges for the surgeon are correctly identifying those patients requiring emergency intervention, especially in particular circumstances (19-24) that include previous administration of analgesics, minor antispasmodics, antisecretory drugs. These are possible effective treatments which may fade clinical signs in patients who have previously undergone several on-call services with incomplete exploration and administered minor therapies. The anamnesis may offer less information for diagnostic in cases with multiple comorbidities, advanced age, neglected, with cognitive deficits (dementia, Alzheimer's). In cases with persistent painful abdomen, with absence of firm imaging information of acute abdomen and relatively normal blood pressure values, an important semiotic value is heart rate $(\mathrm{HR})$ maintained $>90-100$, associated with respiratory frequency of $>18 / \mathrm{min}$.

Tachycardic pulse, increased respiratory rate, suspicion of unconfessed recent trauma can be minor milestones, but sometimes life-saving in the persistence of making a diagnosis as accurate as possible.

SARS-Cov 2 infection-a novel cause of differential diagnosis of acute abdominal pain. The possibility of digestive manifestations in approximately $10 \%$ of cases, due to the binding of SARS-Cov-2 at the level of ACE receptors in the intestinal tract, with fever, abdominal pain, nausea, vomiting, transit disorders, presents with a new element of differential diagnosis on which the on-call surgical team must investigate and exclude (25-38). Moreover, laboratory tests may show an increase in transaminases and amylases in these patients, suggesting hepatic or hepatopancreatic involvement (26-29). Saeed et al (30) conducted a retrospective analysis of all acute abdominal pain cases admitted to their institute and found that 9 out of 70 patients tested positive for SARS-Cov-2, but had no respiratory symptoms. Despite the lack of symptoms, CT scan revealed pulmonary involvement in 6 of them. The mechanisms of abdominal pain during SARS-Cov-2 infection may be classified as pulmonary (with CT abnormalities), due to involvement of lower pulmonary lobes, and extrapulmonary, which can be thrombotic and non-thrombotic (31-35).

The thrombotic causes can be related to the direct invasion of ACE receptors in vascular endothelium, direct viral invasion of the blood vessels, or occlusion resulting from microthrombi formation and include mesenteric vessel ischemia (occlusive or non-occlusive), renal vessel infarcts, and omental infarcts (32). Non-thrombotic causes reported in the literature include pancreatitis, peritonitis, colonic distension, and colitis, and are related to the tropism of the virus to the ACE receptors along the digestive tract (34-38).

Clinical trials conducted during the pandemic revealed unfavorable results, with increased mortality and morbidity in patients who underwent surgery during incubation or early infection with Sars-Cov-2 (1,25,37-39). On the other hand, in the case of certain acute surgical abdomen, and given the high incidence of infections in Bucharest, all patients presenting to the emergency department must be considered suspects until the Covid-19 RT-PCR test comes back negative. This has led both to changes in procedures and patient transport/personnel movement routes, with the limitation of laboratory investigations in the absence of a clear justification. We have also 
observed psycho-behavioral changes, both in patients, and medical staff. The patients often resort to self-medication and delay referral to an outpatient service or hospitalization for fear of possible infection, and present in the emergency room with more severe, complicated forms of disease. On the other hand, the medical personnel, who have to work in uncomfortable protective equipment, under increased psychophysical stress, are tempted to avoid close contact with the possible suspect patient and rely more on imaging and laboratory data, until the absence of SARS-Cov-2 infection is confirmed. The decrease in the number of hospital beds and limited access to specific investigations does not justify the difficulty of diagnosing acute abdomen, but it becomes an obstacle when patients are hospitalized because an accurate diagnosis is difficult to make and for observing patient progress in the following days.

Oncological patients-a vulnerable category during the COVID-19 pandemic. The COVID-19 pandemic has resulted in increased burden on national health systems and cancellation of all non-urgent surgery and oncological activity during the lockdown. This situation has generated a crisis regarding the situation of oncology patients in our country as well as in other European countries (40-42). The limited access to consultations in the primary care system and specialized polyclinics has led to a delay in diagnosing oncological diseases, which explains the higher rate of acute abdomen by stenotic or perforated tumors among the emergency room visits, as an initial presentation of cancer. There was a concern that this vulnerable segment could become a 'silent collateral victim' of the COVID-19 pandemic, as early treatment is essential for achieving prolonged survival (43-46). Reopening the access to adequate care for oncological patients was a priority once elective surgery was reinitiated. These patients are particularly vulnerable to SARS-Cov 2 infection, due to immunosuppression related to neoplasia and related treatments. New guidelines for the therapeutic approach have been established to offer these patients a reasonable time frame for surgery and complementary therapies (40-42).

Diabetic patients-a neglected category during the COVID-19 lockdown. Screening and early diagnosis are the basis for the management of most prevalent chronic diseases, such as cardiovascular disease and diabetes. Studies have shown a dramatic decrease in screening for LDL and HBA1c in the population during the COVID-19 pandemic, concluding that these diseases have also been neglected, with significant consequences for public health (46). The restriction of consultations in specialized outpatient clinics has led to an increase in the incidence of emergencies with decompensated diabetes, including ketoacidosis, which requires a differential diagnosis with acute surgical abdomen. Given on the one hand the long duration of the restrictive measures imposed by the pandemic, but also the fact that diabetes is considered a risk factor for severe evolution in SARS-CoV-2 infection, efforts should be made to further educate people with diabetes regarding the management of their condition, including the use of tele-medicine if possible, educational programs and strategies to ensure medication and glucose testing availability and affordability $(47,48)$.
Anti-COVID 19 measures ease the burden of nosocomial infections. Beneficially, social distancing and frequent hand hygiene and disinfection of health care equipment, supplies and surfaces have resulted in a significant reduction in postoperative infectious complications. No cases of Clostridium infection were recorded in the patients admitted and operated on during the pandemic, and there was only 1 case of wound infection, peritonitis due to cecal tumor perforation, possibly endogenous. This confirms that Lister's principles are still valid, and more concern for asepsis and antisepsis, starting with simple hand washing gestures before and after handling each patient, both for the doctors and the nurses must be maintained after the pandemic.

As the COVID-19 pandemic seems to be still far from over, we should learn to adapt our surgical protocols to the new evidence. Oncological patients are a vulnerable category, neglected in the first months of the pandemic, resulting in a higher rate of emergency room presentations for neoplastic occlusions or perforations. SARS-Cov-2 infection may be a cause of abdominal pain and the differential diagnosis of SARS-Cov-2 in respect to acute abdomen in surgical wards must be considered. Any patients presenting with acute abdomen should be tested and retested for SARS-Cov 2, even in the absence of respiratory symptoms.

Correct wearing of adequate PPE and respecting the strict rules of social distancing, asepsis and antisepsis protocol are urgent and effective for preventing in-hospital transmission of infection both to doctors and medical personnel.

\section{Acknowledgements}

Not applicable.

\section{Funding}

Funding was not received.

\section{Availability of data and materials}

All data generated or analyzed during this study are included in this published article.

\section{Authors' contributions}

SAB, DS, BS, CTa and AMD led the conception and design of this study. CTu, SAB, AMT, DD, AMD and CDB were responsible for the data collection and analysis. SAB, CDB, $\mathrm{BS}, \mathrm{DD}, \mathrm{GV}$ and DS were in charge of drafting the manuscript. DS, CTu, RIS, GV and CTa revised critical perspective for important intellectual content. The final version was read and approved by all the authors.

\section{Ethics approval and consent to participate}

The study was conducted according to the World Medical Association Declaration of Helsinki, using a protocol approved by the local Bioethics Committee from University Emergency Clinical Hospital (Bucharest, Romania). All patients previously signed an informed written consent in regards to hospitalization and investigations. 


\section{Patient consent for publication}

Not applicable

\section{Competing interests}

The authors declare that they have no competing interests.

\section{References}

1. Al-Sadeq DW and Nasrallah GK: The incidence of the novel coronavirus SARS-CoV-2 among asymptomatic patients: A systematic review. Int J Infect Dis 98: 372-380, 2020.

2. Calina D, Docea AO, Petrakis D, Egorov AM, Ishmukhametov AA, Gabibov AG, Shtilman MI, Kostoff R, Carvalho F, Vinceti M, et al: Towards effective COVID-19 vaccines: Updates, perspectives and challenges (Review). Int J Mol Med 46: 3-16, 2020.

3. Papachristou S, Penlioglou T, Stoian AP and Papanas N: COVID-19 and sodium-glucose cotransporter 2 inhibitors: No fear to attempt? Exp Clin Endocrinol Diabetes: Sep 10, 2020 (Epub ahead of print). doi: 10.1055/a-1235-5617.

4. Docea AO, Tsatsakis A, Albulescu D, Cristea O, Zlatian O, Vinceti M, Moschos SA, Tsoukalas D, Goumenou M, Drakoulis N, et al: A new threat from an old enemy: Re-emergence of coronavirus (Review). Int J Mol Med 45: 1631-1643, 2020.

5. Catrinoiu D, Ceriello A, Rizzo M, Serafinceanu C, Montano N, Stoian AP, Udeanu DI, Jinga V, Iorgulescu G and Dumitrescu IB: Diabetes and renin-angiotensin-aldosterone system: Implications for covid-19 patients with diabetes treatment management Farmacia 3: 377-383, 2020.

6. Patel KP, Vunnam SR, Patel PA, Krill KL, Korbitz PM, Gallagher JP, Suh JE and Vunnam RR: Transmission of SARS-CoV-2: An update of current literature. Eur J Clin Microbiol Infect Dis 39: 2005-2011, 2020.

7. Stoian AP, Banerjee Y, Rizvi AA and Rizzo M: Diabetes and the COVID-19 pandemic: How insights from recent experience might guide future management. Metab Syndr Relat Disord 18 : 173-175, 2020

8. Shabbir A, Menon RK, Somani J, So JBY, Ozman M, Chiu PWY and Lomanto D: ELSA recommendations for minimally invasive surgery during a community spread pandemic: A centered approach in Asia from widespread to recovery phases. Surg Endose 34: 3292-3297, 2020

9. Francis N, Dort J, Cho E, Feldman L, Keller D, Lim R Mikami D, Phillips E, Spaniolas K. Tsuda S, et al: SAGES and EAES recommendations for minimally invasive surgery during COVID-19 pandemic. Surg Endosc 34: 2327-2331, 2020.

10. Săftoiu A, Tomulescu V, Tanțău M, Gheorghe C, Dumitru E, Mateescu B, Negreanu L, Jinga M, Seicean A, Ciocîrlan M, et al: SRED-ARCE recommendations for minimally invasive interventions during the covid-19 pandemic in Romania. Chirurgia (Bucur) 115: 289-306, 2020.

11. Englehardt RK, Nowak BM, Seger MV and Duperier FD Contamination resulting from aerosolized fluid during laparoscopic surgery. JSLS 18: e2014.00361, 2014.

12. Serban D, Smarandache CG, Tudor C, Duta LN, Dascalu AM and Alius C: Laparoscopic surgery in COVID-19 era-safety and ethical issues. Diagnostics (Basel) 10: 673, 2020

13. Tran K, Cimon K, Severn M, Pessoa-Silva CL and Conly J: Aerosol generating procedures and risk of transmission of acute respiratory infections to healthcare workers: A systematic review. PLoS One 7: e35797, 2012

14. Tatu AL, Radaschin DS, Constantin VD, Stana $P$ and Ardeleanu V: Laser therapy in superficial morphea lesions-indications, limitations and therapeutic alternatives. J Mind Med Sci 7: 46-51, 2020.

15. Weissman DN, de Perio MA and Radonovich LJ Jr: COVID-19 and risks posed to personnel during endotracheal intubation. JAMA 323: 2027-2028, 2020.

16. Joseph JP,Joseph AO, Oomman S and Jayanthi NVG: Laparoscopic versus open surgery: Aerosols and their implications for surgery during the covid-19 pandemic. Eur Surg: 1-2, Jun 10, 2020 (Epub ahead of print). doi: 10.1007/s10353-020-00644-1.

17. Di Saverio S, Pata F, Khan M, Ietto G, Zani E and Carcano G: Convert to open: The new paradigm for surgery during covid-19? Br J Surg 107: e194, 2020.
18. Ouzzane A and Colin P: Cost-effective filtrating suction to evacuate surgical smoke in laparoscopic and robotic surgery during the covid-19 pandemic. Surg Laparose Endosc Percutan Tech 30: e28-e29, 2020

19. Ravishankaran P, Shah AM and Bhat R: Correlation of interleukin-6, serum lactate, and C-reactive protein to inflammation, complication, and outcome during the surgical course of patients with acute abdomen. J Interferon Cytokine Res 31: 685-690, 2011.

20. Hainaroșie R, Zainea V, Rusescu V, Iana RO, Ghindea T, Suceveanu AP, Stefanescu DC, Ionita IG, Pietroșanu C and Pantea Stoian A: Management of infectious complications in diabetes mellitus mellitus patients. Rom J Mil Med 122: 46-51, 2019.

21. Patterson JW, Kashyap S and Dominique E: Acute Abdomen. In: StatPearls. StatPearls Publishing, Treasure Island, FL, 2020

22. Stoian AP, Toth PP, Kempler P and Rizzo M: Gender differences in the battle against COVID-19: Impact of genetics, comorbidities, inflammation and lifestyle on differences in outcomes. Int J Clin Pract 8: e13666, 2021.

23. Khan MAB and Abu-Zidan FM: Point-of-care ultrasound for the acute abdomen in the primary health care. Turk J Emerg Med 20: $1-11,2020$.

24. Berbece S, Ardeleanu V, Constantin VD, Paunica I and Toma A Therapeutic approach for Amyand's hernia; A case report. J Mind Med Sci 7: 105-109, 2020

25. Li LQ, Huang T, Wang YQ, Wang ZP, Liang Y, Huang TB, Zhang HY, Sun W and Wang Y: COVID-19 patients' clinical characteristics, discharge rate, and fatality rate of meta-analysis. J Med Virol 92: 577-583, 2020.

26. Hadi A, Werge M, Kristiansen KT, Pedersen UG, Karstensen JG, Novovic S and Gluud LL: Coronavirus disease-19 (COVID19) associated with severe acute pancreatitis: Case report on three family members. Pancreatology 20: 665-667, 2020.

27. Kataria S, Sharif A, Ur Rehman A, Ahmed Z and Hanan A: COVID-19 induced acute pancreatitis: A case report and literature review. Cureus 12: e9169, 2020.

28. Ahmed AOE, Mohamed SF, Saleh AO, Al-Shokri SD, Ahmed K and Mohamed MFH: Acute abdomen-like-presentation associated with SARS-CoV-2 infection. IDCases 21: e00895, 2020.

29. Lovece A, Asti E, Bruni B and Bonavina L: Subtotal laparoscopic cholecystectomy for gangrenous gallbladder during recovery from COVID-19 pneumonia. Int J Surg Case Rep 72: 335-338, 2020.

30. Saeed U, Sellevoll HB, Young VS, Sandbaek G, Glomsaker T and Mala T: Covid-19 may present with acute abdominal pain. Br J Surg 107: e186-e187, 2020.

31. Zou X, Chen K, Zou J, Han P, Hao J and Han Z: Single-cell RNA seq data analysis on the receptor ACE2 expression reveals the potential risk of different human organs vulnerable to 2019-nCoV infection. Front Med 14: 185-192, 2020.

32. Bhayana R, Som A, Li MD, Carey DE, Anderson MA, Blake MA Catalano O, Gee MS, Hahn PF, Harisinghani M, et al: Abdominal imaging findings in COVID-19: Preliminary observations. Radiology 297: E207-E215, 2020.

33. Ahmed K and Mohamed MFH: Acute abdomen is not always surgical amid the COVID-19 pandemic. Br J Surg 107: e434-e435, 2020.

34. Kecler-Pietrzyk A, Orsi G, Carthy J and Torreggiani WC: Enteritis and severe abdominal pain as the first presentation of Covid-19. Ir Med J 113: 102, 2020.

35. Suwanwongse K and Shabarek N: Pseudo-appendicitis in an adolescent with COVID-19. Cureus 12: e9394, 2020.

36. Cheung S, Quiwa JC, Pillai A, Onwu C, Tharayil ZJ and Gupta R: Superior mesenteric artery thrombosis and acute intestinal ischemia as a consequence of COVID-19 infection. Am J Case Rep 21: e925753, 2020.

37. Seeliger B, Philouze G, Cherkaoui Z, Felli E, Mutter D and Pessaux P: Acute abdomen in patients with SARS-CoV-2 infection or co-infection. Langenbecks Arch Surg 405: 861-866, 2020.

38. Kielty J, Duggan WP and O'Dwyer M: Extensive pneumatosis intestinalis and portal venous gas mimicking mesenteric ischaemia in a patient with SARS-CoV-2. Ann R Coll Surg Engl 102: e145-e147, 2020.

39. Patriti A, Baiocchi GL, Catena F, Marini P and Catarci M; FACS on behalf of the Associazione Chirurghi Ospedalieri Italiani (ACOI): Emergency general surgery in Italy during the COVID-19 outbreak: First survey from the real life. World J Emerg Surg 15: 36, 2020.

40. Abejón D, Monzón EM, Deer T, Hagedorn JM, Araujo R, Abad C, Rios A, Zamora A and Vallejo R: How to restart the interventional activity in the COVID-19 era. The experience of a private pain unit in Spain. Pain Pract 20: 820-828, 2020. 
41. Qadan M, Hong TS, Tanabe KK, Ryan DP and Lillemoe KD: A multidisciplinary team approach for triage of elective cancer surgery at the massachusetts general hospital during the novel coronavirus COVID-19 outbreak. Ann Surg 272: e20-e21, 2020.

42. Sud A, Jones ME, Broggio J, Loveday C, Torr B, Garrett A Nicol DL, Jhanji S, Boyce SA, Gronthoud F, et al: Collateral damage: The impact on outcomes from cancer surgery of the COVID-19 pandemic. Ann Oncol 31: 1065-1074, 2020.

43. Ardeleanu A, Francu L and Georgescu C: Neoangiogenesis Assessment in esophageal adenocarcinomas. Indian J Surg 77 (Suppl 3): S971-S976, 2015.

44. Sud A, Torr B, Jones ME, Broggio J, Scott S, Loveday C, Garrett A, Gronthoud F, Nicol DL, Jhanji S, et al: Effect of delays in the 2-week-wait cancer referral pathway during the COVID-19 pandemic on cancer survival in the UK: A modelling study. Lancet Oncol 21: 1035-1044, 2020.
45. Tulin A, Slavu I, Tulin R, Alecu L, Jecan CR, Orlov C, Iaciu CI, Stanculeanu DL, Hainarosie R, Pituru S, et al: Does sex of the patient play a role in survival for MSI colorectal cancer? J Mind Med Sci 5: 101-108, 2018.

46. Wright A, Salazar A, Mirica M, Volk LA and Schiff GD: The invisible epidemic: Neglected chronic disease management during COVID-19. J Gen Intern Med 35: 2816-2817, 2020.

47. Scott ES, Jenkins AJ and Fulcher GR: Challenges of diabetes management during the COVID-19 pandemic. Med J Aust 213: 56-57.e1, 2020.

48. Caballero AE, Ceriello A, Misra A, Aschner P, McDonnell ME, Hassanein M, Ji L, Mbanya JC and Fonseca VA: COVID-19 in people living with diabetes: An international consensus. J Diabetes Complications 34: 107671, 2020. 